\title{
Photonic Implementation of Quantum Relay and Encoders/Decoders for Sparse-Graph Quantum Codes Based on Optical Hybrid
}

\author{
Ivan B. Djordjevic, Member, IEEE
}

\begin{abstract}
We show that any family of deterministic universal quantum gates can be implemented in integrated optics. We further show how to implement Pauli gates, needed in quantum-error correction, using the same technology. Two basic circuits required in quantum teleportation systems are Bell states and quantum relay. We describe how to implement them in integrated optics. Finally, we study the implementation of sparse-graph (SG) quantum codes in integrated optics. We demonstrate that entanglement assisted SG codes significantly outperform corresponding dual-containing quantum codes.
\end{abstract}

Index Terms-Integrated optics devices, quantum communications, quantum information and processing (QIP).

\section{INTRODUCTION}

$\mathbf{Q}$ UANTUM information processing (QIP) is an exciting research area with numerous applications including quantum key distribution (QKD), quantum teleportation, quantum computing, quantum networks, quantum lithography, and quantum memories. To perform an arbitrary quantum computation operation, a minimum number of gates, known as universal quantum gates [1], is needed. The most popular sets of universal quantum gates are: 1) \{Hadamard $(\mathrm{H})$, phase $(\mathrm{S}), \pi / 8$ (T), Controlled-NOT (CNOT) $\}$ gates; 2$)\{\mathrm{H}, \mathrm{S}, \mathrm{CNOT}$, Toffoli $\left.\left(\mathrm{U}_{\mathrm{T}}\right)\right\}$ gates; 3$)$ \{Barenco\} gate [1]; and 4) \{Deutsch\} gate [1]. In this letter, we show that all these sets of universal quantum gates can be implemented in integrated optics by using either Mach-Zehnder interferometer (MZI) or optical hybrid (OH) and highly nonlinear fiber (HNLF) as basic building blocks. The basic quantum circuit needed in the quantum teleportation system is the Bell states preparation circuit. Because it is based on CNOT and Hadamard gates it can implemented using the same technology. To extend the transmission distance of current quantum teleportation systems, the implementation of quantum relay is of crucial importance. Because this circuit is based on Bell states preparation circuit, controlled- $X$ gate and controlled-Z gates, it can also be implemented in integrated optics. The QIP relies on delicate superposition states that are sensitive to the interaction with environment, resulting in quantum errors. Moreover, the quantum gates are imperfect and the use of quantum error correction coding (QECC) is essential

Manuscript received February 13, 2010; revised July 04, 2010; accepted July 17, 2010. Date of publication July 23, 2010; date of current version September $10,2010$.

The author is with the Electrical and Computer Engineering Department, University of Arizona, Tucson, AZ 85721 USA (e-mail: ivan@ece.arizona.edu).

Color versions of one or more of the figures in this letter are available online at http://ieeexplore.ieee.org.

Digital Object Identifier 10.1109/LPT.2010.2060477

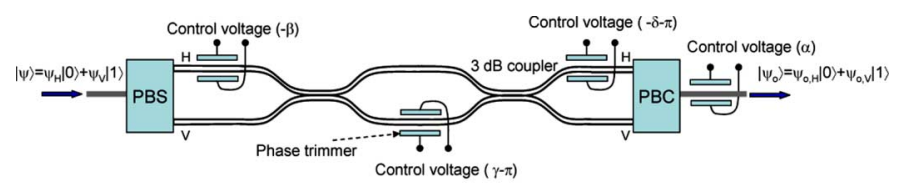

(a)

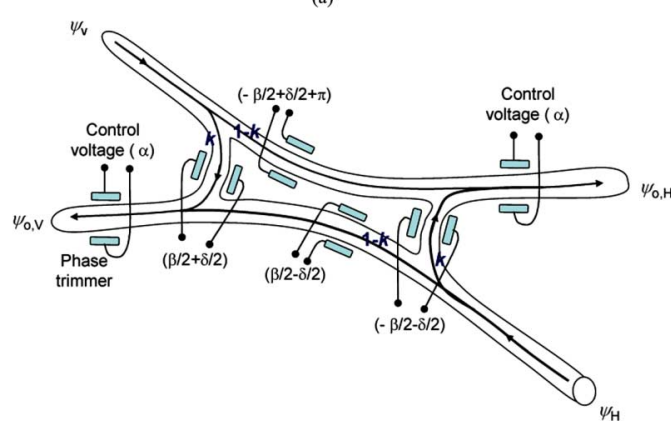

(b)

Fig. 1. Implementation of arbitrary single-qubit quantum gate in integrated optics based on single: (a) Mach-Zehnder interferometer and (b) optical hybrid. PBS/C: polarization beam splitter/combiner. ( $\alpha, \beta$ and $\delta$ are phase shifts introduced by corresponding phase trimmers).

to enable the fault-tolerant computing [1]-[3] and to deal with quantum errors. The QECC encoders/decoders are essentially based on Pauli gates. We show how to implement the Pauli operators, important in QECC, in integrated optics by using MZIs/OHs and HNLF as well. We also show how to implement "sparse" quantum encoders and decoders [2], in integrated optics. We restrict our attention to sparse-graph quantum codes [2], because their quantum check matrices are sparse so that a small number of interactions is needed in corresponding syndrome operators.

\section{UNIVERSAL QUANTUM GATES IN INTEGRATED OPTICS}

In what follows, the logical " 0 " is represented by a horizontal (H) photon $|0\rangle \equiv|H\rangle$ and the logical " 1 " is represented by a vertical $(\mathrm{V})$ photon $|1\rangle \equiv|V\rangle$. An arbitrary single-qubit gate can be implemented in integrated optics based on MZI or OH as shown in Fig. 1. We use a polarization beam splitter (PBS) at the input of quantum gate and a polarization beam combiner (PBC) at the output of the gate. The horizontal output (input) of PBS (PBC) is denoted by $\mathrm{H}$, while the vertical output (input) of PBS (PBC) is denoted by $\mathrm{V}$. The input qubit is denoted by $|\psi\rangle=\psi_{\mathrm{H}}|0\rangle+\psi_{\mathrm{V}}|1\rangle=\left[\begin{array}{ll}\psi_{\mathrm{H}} & \psi_{\mathrm{V}}\end{array}\right]^{\mathrm{T}}$, while the output qubit is denoted by $\left|\psi_{\mathrm{o}}\right\rangle=\psi_{\mathrm{o}, \mathrm{H}}|0\rangle+\psi_{\mathrm{o}, \mathrm{V}}|1\rangle=\left[\psi_{\mathrm{o}, \mathrm{H}} \psi_{\mathrm{o}, \mathrm{V}}\right]^{\mathrm{T}}$. In Fig. 1(a), we show an implementation based on single MZI; while in Fig. 1(b) we show an implementation based on single $\mathrm{OH}$. For convenience, the power splitting ratio $k$ of $\mathrm{OH}$ is parameterized as follows: $k=\cos ^{2}(\gamma / 2)$. In both schemes, 
the output qubit is related to the input qubit by

$$
\begin{aligned}
& {\left[\begin{array}{l}
\psi_{\mathrm{o}, \mathrm{H}} \\
\psi_{\mathrm{o}, \mathrm{V}}
\end{array}\right]=U\left[\begin{array}{l}
\psi_{\mathrm{H}} \\
\psi_{\mathrm{V}}
\end{array}\right]} \\
& U=\left[\begin{array}{lc}
\cos \left(\frac{\gamma}{2}\right) e^{j(\alpha-\beta / 2-\delta / 2)} & -\sin \left(\frac{\gamma}{2}\right) e^{j(\alpha-\beta / 2+\delta / 2)} \\
\sin \left(\frac{\gamma}{2}\right) e^{j(\alpha+\beta / 2-\delta / 2)} & \cos \left(\frac{\gamma}{2}\right) e^{j(\alpha+\beta / 2+\delta / 2)}
\end{array}\right] .
\end{aligned}
$$

The $U$-matrix in (1) represents the matrix representation of an arbitrary single-qubit quantum gate according to the Z-Y decomposition theorem. For $\mathrm{OH}$, the corresponding phase shifts $\alpha, \beta, \delta$ can be introduced by phase trimmer either thermally or electrooptically, while the proper power splitting ratio $k=$ $\cos ^{2}(\gamma / 2)$ should be set in fabrication phase. By setting $\gamma=$ $\delta=0$ rad, $\alpha=\pi / 4$ and $\beta=\pi / 2$ rad $U$-gate described by (1) operates as the phase gate; by setting $\gamma=\delta=0 \mathrm{rad}, \alpha=\pi / 8$ and $\beta=\pi / 4 \mathrm{rad}$, the $U$-gate operates as $\pi / 8$ gate; while by setting $\gamma=\pi / 2, \alpha=\pi / 2, \beta=0 \mathrm{rad}$ and $\delta=\pi$, the $U$-gate given by (1) operates as Hadamard gate. To complete the implementation of set 1) of universal quantum gates, the implementation of CNOT-gate is needed. The authors in [4] proposed the use of directional couplers to implement the CNOT-gate. However, the control output qubit $\left[c_{\mathrm{H}, \mathrm{o}}, c_{\mathrm{V}, \mathrm{o}}\right]^{\mathrm{T}}$ is related to the input control qubit $\left[c_{\mathrm{H}}, c_{\mathrm{V}}\right]^{\mathrm{T}}$ and input target qubit $\left(t_{\mathrm{H}}, t_{\mathrm{V}}\right)^{\mathrm{T}}$ by [4]

$$
\left[c_{\mathrm{H}, \mathrm{o}}=\left(\frac{1}{\sqrt{3}}\right)\left(\sqrt{2} v_{c}+c_{\mathrm{H}}\right), c_{\mathrm{V}, \mathrm{o}}=\left(\frac{1}{\sqrt{3}}\right)\left(-c_{\mathrm{V}}+t_{\mathrm{H}}+t_{\mathrm{V}}\right)\right]^{\mathrm{T}}
$$

which violates CNOT-gate operation definition [1]. In Fig. 2(a), we show the deterministic implementation of the CNOT-gate based on the single-qubit gate shown in Fig. 1 and HNLF. It can be shown that output control $\left|C_{\mathrm{o}}\right\rangle=\left[c_{\mathrm{H}, \mathrm{o}} c_{\mathrm{V}, \mathrm{o}}\right]^{\mathrm{T}}$ and target qubits $\left|T_{\mathrm{o}}\right\rangle=\left[t_{\mathrm{H}, \mathrm{o}} t_{\mathrm{V}, \mathrm{o}}\right]^{\mathrm{T}}$ are related to corresponding input qubits by

$$
\begin{aligned}
& {\left[\begin{array}{c}
c_{\mathrm{H}, \mathrm{o}} \\
c_{\mathrm{V}, \mathrm{o}} \\
t_{\mathrm{H}, \mathrm{o}} \\
t_{\mathrm{V}, \mathrm{o}}
\end{array}\right]=\frac{1}{\sqrt{2}} \underbrace{\left[\begin{array}{cccc}
1 & 1 & 0 & 0 \\
1 & -1 & 0 & 0 \\
0 & 0 & 1 & 1 \\
0 & 0 & 1 & -1
\end{array}\right]}_{I \otimes H} \underbrace{\left[\begin{array}{cccc}
1 & 0 & 0 & 0 \\
0 & 1 & 0 & 0 \\
0 & 0 & 1 & 0 \\
0 & 0 & 0 & -1
\end{array}\right]}_{K}} \\
& \times \frac{1}{\sqrt{2}} \underbrace{\left[\begin{array}{cccc}
1 & 1 & 0 & 0 \\
1 & -1 & 0 & 0 \\
0 & 0 & 1 & 1 \\
0 & 0 & 1 & -1
\end{array}\right]}_{I \otimes H}\left[\begin{array}{c}
c_{\mathrm{H}} \\
c_{\mathrm{V}} \\
t_{\mathrm{H}} \\
t_{\mathrm{V}}
\end{array}\right] \\
& =U_{C N O T}\left[\begin{array}{c}
c_{\mathrm{H}} \\
c_{\mathrm{V}} \\
t_{\mathrm{H}} \\
t_{\mathrm{V}}
\end{array}\right], U_{C N O T}=\left[\begin{array}{llll}
1 & 0 & 0 & 0 \\
0 & 1 & 0 & 0 \\
0 & 0 & 0 & 1 \\
0 & 0 & 1 & 0
\end{array}\right] \text {. }
\end{aligned}
$$

The Kerr nonlinearity device in Fig. 2(a) performs the controlled- $Z$ operation. In the absence of control $c_{\mathrm{V}}$-photon, the target qubit is unaffected because $H^{2}=I$ (identity operator). In the presence of control $c_{\mathrm{V}}$-photon, thanks to the cross-phase modulation in HNLF, the target vertical photon experiences the phase shift $\chi L$, where $\chi$ is the third-order nonlinearity susceptibility coefficient and $L$ is the HNLF length. By selecting appropriately the fiber length we obtain $\chi L=\pi$ and the overall action on target qubit is $H Z H=X$, which corresponds to the CNOT gate action. It has recently been demonstrated by Matsuda et al. [5] that Kerr nonlinearity can introduce the cross-phase modulation phase shift at single-photon level by using photonic crystal fibers. Unfortunately, the introduced phase shift was rather small

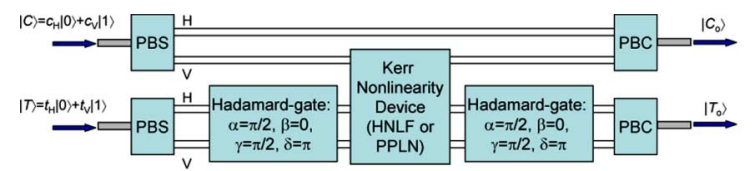

(a)

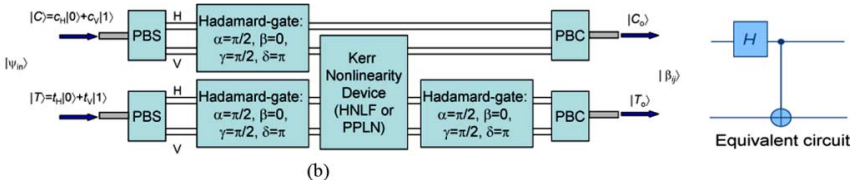

Fig. 2. Photonic implementation of: (a) CNOT gate and (b) Bell state preparation circuit. PPLN: periodically poled LiNbO3.

$\left(9.8 \times 10^{-8} \mathrm{rad}\right.$ for fiber length $\left.4.7 \mathrm{~m}\right)$. On the other hand, the nonlinear Kerr phase shift up to $\pi / 4$ at the single-photon has been demonstrated in [6] by using the cavity quantum electrodynamics (CQED)-based devices. Given the fact that this gate behaves as imperfect CNOT-gate for $\chi L \in(\pi / 2, \pi)$, the deterministic CNOT-gate might become reality soon. The imperfect CNOT-gate will fail with certain probability and the use of QECC is essential to provide fault-tolerant computing. The different implementations of CNOT-gate in [4], [8] employ multi-rail/dual-rail representation, and as such cannot be implemented at all using conventional single-mode integrated optics. This is a key disadvantage of multi-rail representation compared to our proposal. Notice that both deterministic and probabilistic CNOT-gates, can be used to implement proposed quantum relay and sparse-graph encoders/decoders. One such CNOT gate, based on Sagnac interferometer in the telecommunication band $(\sim 1550 \mathrm{~nm})$, has been recently reported by Chen et al. in [7]. Moreover, the proposed quantum relay and SG encoder/decoder architectures are universal and implementable in any of currently available technologies.

In the reminder of this section, we describe the implementation of Pauli gates $\mathrm{X}, \mathrm{Y}$, and $\mathrm{Z}$ in integrated optics. By using the $U$-gate shown in Fig. 1 and by appropriately setting the phase shifts $\alpha, \beta, \gamma$, and $\delta$ we can obtain the corresponding Pauli gates. The $Y$-gate is obtained by setting $\gamma=\pi, \beta=\delta=0 \mathrm{rad}$, and $\alpha=\pi / 2$; the $Z$-gate is obtained by setting $\gamma=\delta=0 \mathrm{rad}$, $\alpha=\pi / 2$, and $\beta=\pi$; and the $X$-gate is obtained by setting $\gamma=\pi, \delta=0 \mathrm{rad}, \alpha=\pi / 2$, and $\beta=-\pi$.

\section{Photonic Implementation of Quantum Relay}

In this section, we first describe the implementation of Bell states preparation circuit in integrated optics, required in quantum teleportation systems, which is shown in Fig. 2(b). Among many possible versions of Hadamard and CNOT-gates we have chosen two with similar propagation times. The upper circuit operates as Hadamard gate, while the rest of the circuit operates as CNOT gate as already explained in description of Fig. 2(a). It can be shown that output quantum state $\left|\beta_{i j}\right\rangle$ is related to the input state $\left|\psi_{\text {in }}\right\rangle$ by:

$$
\begin{aligned}
\left|\beta_{i j}\right\rangle & =\frac{1}{\sqrt{2}}\left[\begin{array}{llll}
1 & 0 & 0 & 0 \\
0 & 1 & 0 & 0 \\
0 & 0 & 0 & 1 \\
0 & 0 & 1 & 0
\end{array}\right]\left[\begin{array}{cccc}
1 & 0 & 1 & 0 \\
0 & 1 & 0 & 1 \\
1 & 0 & -1 & 0 \\
0 & 1 & 0 & -1
\end{array}\right]\left|\psi_{i n}\right\rangle \\
& =\frac{1}{\sqrt{2}}\left[\begin{array}{l}
c_{\mathrm{H}} t_{\mathrm{H}}+c_{\mathrm{V}} t_{\mathrm{V}} \\
c_{\mathrm{H}} t_{\mathrm{V}}+c_{\mathrm{V}} t_{\mathrm{V}} \\
c_{\mathrm{V}} t_{\mathrm{H}}-c_{\mathrm{V}} t_{\mathrm{V}} \\
c_{\mathrm{H}} t_{\mathrm{H}}-c_{\mathrm{V}} t_{\mathrm{H}}
\end{array}\right] .
\end{aligned}
$$




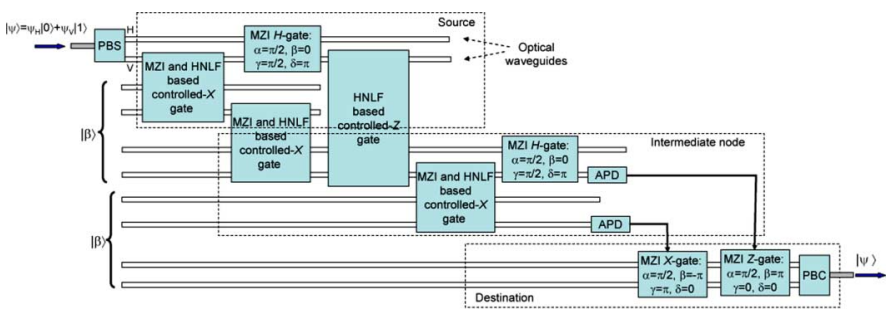

Fig. 3. Photonic implementation of quantum relay.

For example, by setting $c_{\mathrm{H}}=t_{\mathrm{H}}=1$ and $c_{\mathrm{V}}=t_{\mathrm{V}}=$ 0 we obtain the Bell state $\left|\beta_{00}\right\rangle=\left[\begin{array}{llll}1 & 0 & 0 & 1\end{array}\right]^{\mathrm{T}} / \sqrt{2}=$ $(|00\rangle+|11\rangle) / \sqrt{2}$. In Fig. 3, we describe how to implement the quantum relay based on Bell states preparation circuit [shown in Fig. 2(b)], Hadamard, controlled $-X$ and controlled $-Z$ gates described above. We employ the principle of differed measurement and perform corresponding measurements only in last intermediate node. The measurements circuits in Fig. 3 represent the avalanche photodiodes (APDs), which are used to detect the presence of $c_{\mathrm{V}}$-photons in corresponding control qubits. The detection of $c_{\mathrm{V}}$-photons triggers the application of required control voltages on phase trimmers to perform controlled- $X$ and controlled- $Z$ operation at the destination node.

\section{Photonic Implementation of SG ENCODERS/Decoders}

Because the photon polarization is a fragile recourse of quantum information, in this section, we discuss the sparse-graph encoder/decoder implementation in integrated optics. Most popular quantum codes belong to the class of dual-containing Calderbank-Shor-Steane (CSS) codes [1], with (quantum) check matrix represented by

$$
A=\left[\begin{array}{c|c}
\boldsymbol{H} & 0 \\
0 & H
\end{array}\right]
$$

where $\boldsymbol{H} \boldsymbol{H}^{\mathrm{T}}=0$, which is equivalent to $C^{\perp}(\boldsymbol{H}) \subset C(\boldsymbol{H})$, where $C(\boldsymbol{H})$ is the code having $\boldsymbol{H}$ as the parity check matrix, and $C^{\perp}(\boldsymbol{H})$ is its corresponding dual code. The quantum LDPC codes have many advantages over other classes of quantum codes, thanks to the sparseness of their parity-check matrices [2]. For example, for

$$
H=\left[\begin{array}{llllll}
1 & 0 & 0 & 1 & 1 & 1 \\
1 & 1 & 1 & 0 & 0 & 1 \\
0 & 1 & 1 & 1 & 1 & 0
\end{array}\right]
$$

by performing Gauss-Jordan elimination the quantum check matrix (5) can be put in standard form

$$
\boldsymbol{A}=\left[\begin{array}{llllllllllll}
1 & 0 & 0 & 1 & 1 & 1 & 0 & 0 & 0 & 0 & 0 & 0 \\
0 & 1 & 1 & 1 & 1 & 0 & 0 & 0 & 0 & 0 & 0 & 0 \\
0 & 0 & 0 & 0 & 0 & 0 & 1 & 1 & 1 & 0 & 0 & 1 \\
0 & 0 & 0 & 0 & 0 & 0 & 1 & 0 & 0 & 1 & 1 & 1
\end{array}\right] .
$$

It can be shown based on [1] that encoding and decoding circuits require only Hadamard and CNOT gates, whose implementation in integrated optics is already discussed in Section II. The main drawback of dual-containing LDPC codes is the fact that they are essentially girth-4 codes, which do not perform well under sum-product algorithm (commonly used in decoding of LDPC codes). On the other hand, it was shown in [3] that through the use of entanglement arbitrary classical codes can be used in correction of quantum errors, not only girth-4 codes. The number of entanglement qubits (ebits) needed in EA LDPC codes is $e=\operatorname{rank}\left(\boldsymbol{H} \boldsymbol{H}^{\mathrm{T}}\right)$ (where $\boldsymbol{H}$ is the parity-check ma-

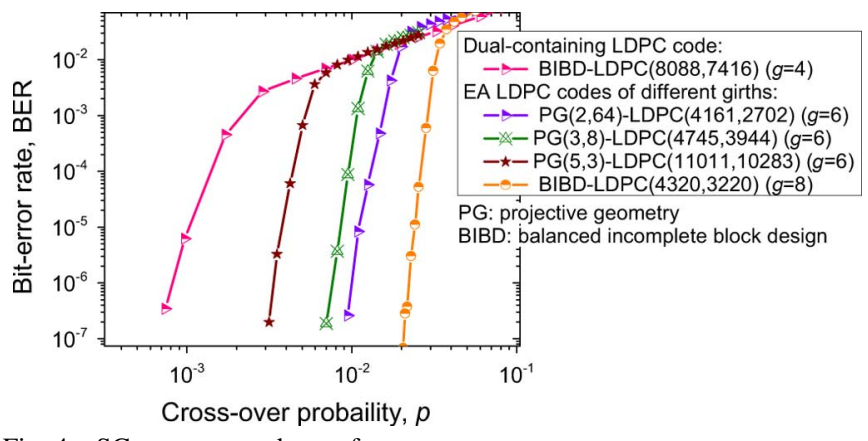

Fig. 4. SG quantum codes performance.

trix of a classical code and $\operatorname{rank}($.$) is the rank of a given ma-$ trix) so that minimum number of required Bell states is one. For example, a classical code given by $\boldsymbol{H}$-matrix below has rank $\left(\boldsymbol{H} \boldsymbol{H}^{\mathrm{T}}\right)=1$ and girth 6

$$
H=\left[\begin{array}{llllll}
1 & 0 & 1 & 0 & 1 & 0 \\
0 & 1 & 0 & 1 & 1 & 0 \\
1 & 0 & 0 & 1 & 0 & 1 \\
0 & 1 & 1 & 0 & 0 & 1
\end{array}\right]
$$

and corresponding EA code requires only one ebit to be shared between source and destination, which is a small increase in complexity compared to dual-containing codes. Since arbitrary classical codes can be used with this approach, including LDPC codes of girth $g \geq 6$, the performance of quantum LDPC codes can significantly be improved. For dual containing code rank $\left(\boldsymbol{H H}^{\mathrm{T}}\right)=0$ so that entanglement between source and destination is not needed. Unfortunately, dual-containing codes are girth-4 codes. It is well known that the iterative LDPC decoding steps are independent up to $g / 2$ iterations. By increasing the girth we therefore improve the overall BER performance. Because two Pauli operators on $n$-qubits commute if and only if there is an even number of positions in which they differ (neither of which is the identity $I$ operator), we can extend the generators in $\boldsymbol{A}$ (for $\boldsymbol{H}$ ) by adding $e$ columns ( $e=1$ in example above) so that they can be embedded into a larger Abelian group; the procedure is known as Abelianization in abstract algebra. In Fig. 4, we provide comparison of EA LDPC codes of girth $g=6$ and 8 against a dual-containing LDPC code $(g=4)$. We see that EA LDPC codes outperform for more than an order in magnitude of a corresponding dual-containing code.

\section{REFERENCES}

[1] F. Gaitan, Quantum Error Correction and Fault Tolerant Quantum Computing. Boca Raton, FL: CRC, 2008.

[2] D. J. C. MacKay, G. Mitchison, and P. McFadden, "Sparse-Graph codes for quantum error correction," IEEE Trans. Inf. Theory, vol. 50, no. 9, pp. 2315-2330, Sep. 2004.

[3] T. Brun, I. Devetak, and M.-H. Hsieh, "Correcting quantum errors with entanglement," Science, vol. 314, pp. 436-439, 2006.

[4] T. C. Ralph, N. K. Langford, T. B. Bell, and A. G. White, "Linear optical Controlled-NOT gate in the coincidence basis," Phys. Rev. A, vol. 65, pp. 062324-1-062324-5, 2002.

[5] N. Matsuda, R. Shimizu, Y. Mitsumori, H. Kosaka, and K. Edamatsu, "Observation of optical-fibre Kerr nonlinearity at the single-photon level," Nature Photon., vol. 3, pp. 95-98, 2009.

[6] I. Fushman, D. Englund, A. Faraon, N. Stoltz, P. Petroff, and J. Vuckovic, "Controlled phase shifts with a single quantum dot," Science, vol. 320, no. 5877, pp. 769-772, 2008.

[7] J. Chen et al., "Demonstration of a quantum Controlled-NOT gate in the telecommunications band," Phys. Rev. Lett, vol. 100, p. 133603, 2008.

[8] E. Knill, R. Laflamme, and G. J. Milburn, "A scheme for efficient quantum computation with linear optics," Nature, vol. 409, pp. 46-52, 2001. 